Bull. Austral. Math. Soc.

$47 \mathrm{~A} 05,46 \mathrm{~B} 15,47 \mathrm{~B} 07$

VoL. 68 (2003) [13-20]

\title{
A RIEMANN TYPE THEOREM FOR SERIES OF OPERATORS ON BANACH SPACES
}

\author{
A. Aizpuru, A. Gutiérrez-Dávila and A. Sala
}

We study Kalton's theorem on the unconditional convergence of series of compact operators and we use some matrix techniques to obtain sufficient conditions, weaker than previous ones, on the convergence and unconditional convergence of series of compact operators. Finally, we characterise weak unconditionally Cauchy series in $C \mathcal{L}(X, Y)$ in terms of certain spaces of vector sequences.

\section{INTRODUCTION}

Let $X, Y$ be two Banach spaces. We denote by $C \mathcal{L}(X, Y)$ (respectively $K(X, Y)$ ) the Banach space of bounded (respectively, compact) linear maps from $X$ to $Y$. Kalton [8] proved that if $\sum_{i} T_{i}$ is subseries convergent in $K(X, Y)$, with respect to the weak operator topology, and $X^{*}$ contains no subspace isomorphic to $l_{\infty}$, then $\sum_{i} T_{i}$ is subseries convergent with respect to the norm topology of $K(X, Y)$. Kaftal and Weiss [7] proved that if $H$ is a Hilbert space and $\left(T_{n}\right)_{n}$ is a sequence in $K(H)$ such that

(i) $\sum_{n} T_{n}$ is unconditionally convergent for the stronger operator topology, and

(ii) for every $F \subset \mathbb{N}$ the operator $\sum_{n \in F} T_{n}$ is compact, then $\sum_{n} T_{n}$ is unconditionally convergent in ${ }^{n \in F} K(H)$.

Qingying $\mathrm{Bu}$ and Congxin $\mathrm{Wu}$ [5] proved the following result, which is equivalent to the previous result of Kalton. Every series $\sum_{i} K_{i}$ of operators in $C \mathcal{C}(X, Y)$ such that:

(i) $\sum_{i} K_{i}$ converges unconditionally in the weak operator topology (in the proof the series $\sum_{i} K_{i}$ should be subseries convergent with respect to the weak operator topology), and

(ii) $\sum_{i \in F} K_{i}$ is a compact operator for every index set $F \subset \mathbb{N}$, is unconditionally convergent in the norm if and only if $X^{*}$ contains no copy of $c_{0}$.

In this paper, we use some matrix techniques $([2,3,4])$ to improve the above mentioned results.

Received 14th August, 2002

Copyright Clearance Centre, Inc. Serial-fee code: 0004-9727/03 \$A2.00+0.00. 
We shall say that $\mathcal{F}$ is a natural family if $\mathcal{F} \subset \mathcal{P}(\mathbb{N})$ and $\mathcal{F}$ contains the family $\phi_{0}(\mathbb{N})$ of the finite subsets of $\mathbb{N}$. We also say that $\mathcal{F}$ has the property $S_{1}$ if for any pair $\left[\left(A_{i}\right),\left(B_{i}\right)\right]$ of mutually disjoint sequences of disjoint subsets of $\phi_{0}(\mathbb{N})$ there exist $B \in \mathcal{F}$ and an infinite set $M \subset \mathbb{N}$ such that $A_{i} \subset B$ and $B_{i} \subset B^{c}$. We shall say that the family $\mathcal{F}$ is subsequentially complete if for any sequence $\left(A_{i}\right)_{i}$ of disjoint sets in $\mathcal{F}$ there exist an infinite set $M \subset \mathbb{N}$ and $B \in \mathcal{F}$ such that $B=\bigcup_{i \in M} A_{i}$.

We must recall that any atomic subalgebra $\mathcal{F}$ of $\mathcal{P}(\mathbb{N})$ is natural.

Let $X$ be a vector space and let $T$ be a vector space topology on $X$. Let $\sum_{i} x_{i}$ be a series in $X$ and let $\mathcal{F}$ be a natural family. We shall say that $\sum x_{i}$ is $\mathcal{F}-T$ convergent if for every $A \in \mathcal{F}$ the series $\sum_{j \in A} x_{j}$ converges in $(X, T)$. It can be proved ([2]) that if $\mathcal{F}$ is a natural family with the property $S_{1}$ and $\sum x_{i}$ is a $\mathcal{F}$-weak convergent series in a Banach $X$ then $\sum_{i} x_{i}$ is unconditionally convergent. The following result can also be proved ([2]) (we shall denote it as (1) throughout this paper). Let $\left(x_{i j}\right)_{i, j}$ be a matrix in a normed space $X$ such that $\left(x_{i j}\right)_{i}$ is a Cauchy sequence for $j \in \mathbb{N}$, and for $i \in \mathbb{N}$ the series $\sum_{j} x_{i j}$ is subseries convergent. Then the following conditions are equivalent: (1) there exists a natural family $\mathcal{F}$ with the property $S_{1}$ such that $\left(\sum_{j \in B} x_{i j}\right)_{i}$
is Cauchy, for $B \in \mathcal{F}$;

(2) the sequence $\left(\sum_{j \in A_{n}} x_{i j}\right)_{i}$ is Cauchy uniformly in $n \in \mathbb{N}$, for every disjoint sequence $\left(A_{n}\right)_{n}$ of $\phi_{0}(\mathbb{N})$.

In this paper we obtain several results on the convergence and unconditional convergence of series of compact operators. The results that appear in $([7,8,9])$ can be obtained as an immediate consequence. Our results are based on the $\mathcal{F}$-convergence of series, where $\mathcal{F}$ denotes a natural family or a natural Boolean algebra with an appropriate property. We also obtain an interesting characterisation of weak unconditionally Cauchy series in $C \mathcal{L}(X, Y)$.

\section{CONVERGENCE OF SERIES OF COMPACT OPERATORS}

THEOREM 2.1. Let $X, Y$ be two Banach spaces and let $\mathcal{F}$ be a natural family with the property $S_{1}$. Let $\left(T_{i}\right)_{i}$ be a sequence of compact operators from $X$ to $Y$. The series $\sum_{i} T_{i}$ is unconditionally convergent in $K(X, Y)$ if and only if $\sum_{i} T_{i}^{*}$ is $\mathcal{F}$ weak operator topology convergent, and for any $B \in \mathcal{F}$ the operator defined by $T_{B}^{*}(g)$ $=w \sum_{j \in B} T_{j}^{*} g$ is compact.

Proof: If $x \in X$ then the series $\sum_{j} T_{j} x$ is $\mathcal{F}$-weak convergent and, therefore, is unconditionally convergent. We also have that, for $g \in Y^{*}, \sum_{j} T_{j}^{*} g$ is unconditionally convergent in $X^{*}$.

Since the rank of a compact operator is separable, we can suppose that $Y$ is separable. Let us suppose that $\sum_{j} T_{j}$ is not unconditionally convergent in $C \mathcal{L}(X, Y)$. In this case, 
there exist $\varepsilon>0$ and a sequence $\left(F_{k}\right)_{k}$ in $\phi_{0}(\mathbb{N})$ such that $\left\|\sum_{j \in F_{k}} T_{j}\right\|>\varepsilon$ and $\sup F_{k}$ $<\inf F_{k+1}$ for $k \in \mathbb{N}$. Since $\left\|\sum_{j \in F_{k}} T_{j}^{*}\right\|>\varepsilon$, for every $k \in \mathbb{N}$ there exists $g_{k} \in B_{Y} \cdot$ such that $\left\|\sum_{j \in F_{k}} g_{k} T_{j}\right\|>\varepsilon$. There exists a subsequence of $\left(g_{k}\right)_{k}$ that is weak $k^{*}$ convergent to some $g_{0} \in B_{Y^{*}}$. Without loss of generality we shall suppose that $w^{*} \lim _{k} g_{k}=g_{0}$.

Let us consider the matrix $\left(g_{i} T_{j}\right)_{i, j}$. We have that:

(1) Since, for $j \in \mathbb{N}, T_{j}$ is compact, the sequence $\left(g_{i} T_{j}\right)_{i}$ is convergent.

(2) The sequence $\left(\sum_{j \in B} g_{i} T_{j}\right)_{i}$ is convergent in $X^{*}$; therefore, the matrix $\left(g_{i}, T_{j}\right)_{i, j}$ satisfies (1) (see introduction). This contradicts that $\left\|\sum_{j \in F_{k}} g_{i} T_{j}\right\|>\varepsilon$ for
$i \in \mathbb{N}$.

REMARK. Let us consider in $K(X, Y)$ the topology $T_{w^{\prime}}$ defined as follows: a net $\left(T_{\alpha}\right)_{\alpha \in I}$ has $w^{\prime} \lim _{\alpha} T_{\alpha}=T_{0}$ if and only if $\lim _{\alpha} T_{\alpha}^{*}=T_{0}^{*}$ for weak operator topology. It can be proved ([7]) that if $\left(T_{n}\right)_{n}$ is a sequence in $K(X, Y)$ such that $w^{\prime} \lim _{n} T_{n}=T_{0}$ and $T_{0} \in K(X, Y)$ then $\lim T_{n}=T_{0}$ for the weak topology in $K(X, Y)$. From this result, we obtain that, under the hypothesis of Theorem $2.1, \sum_{i} T_{i}$ is $\mathcal{F}$-weak convergent, and therefore $\sum_{i} T_{i}$ is unconditionally convergent.

COROLLARY 2.2. Let $X, Y$ be two Banach spaces and let $\mathcal{F}$ be a natural family with the property $S_{1}$. Let $\left(T_{i}\right)_{i}$ be a sequence of compact operators from $X$ to $Y$ and let us suppose that $X^{*}$ does not have a copy of $l_{\infty}$. Then, the series $\sum_{i} T_{i}$ is unconditionally convergent in $K(X, Y)$ if and only if $\sum_{j} T_{j}$ is $\mathcal{F}$-weak operator topology convergent and, for $B \in \mathcal{F}$, the operator defined by $T_{B}(x)=\sum_{j \in B} T_{j} x$ is compact.

Proof: For $x \in X$, the series $\sum_{j} T_{j} x$ is unconditionally convergent. Hence, for $g \in Y^{*}$, we have that $\sum_{j} g\left(T_{j} x\right)$ is unconditionally convergent. This proves that $\sum_{j} g T_{j}$ is weak unconditionally of Cauchy. Since $X^{*}$ does not have a copy of $c_{0}, \sum_{j} g T_{j}$ is unconditionally convergent in $X^{*}$. Now, it is sufficient to proceed as in the proof of Theorem 2.1.

The Vitali-Hahn-Saks property for a Boolean algebra is related to classical Measure Theory ([10]). In the hypothesis of our next corollary, we consider a natural Boolean algebra $\mathcal{F}$ with the Vitali-Hahn-Saks property instead of a natural family $\mathcal{F}$ with the property $S_{1}$.

Corollary 2.3. Let $X, Y$ be two Banach spaces and let $\mathcal{F}$ be a natural Boolean algebra with the Vitali-Hahn-Saks property. Let $\left(T_{i}\right)_{i}$ be a sequence of compact operators from $X$ to $Y$. Then,

(a) $\sum_{j} T_{j}$ is unconditionally convergent if and only if $\sum_{j \in B} T_{j}^{*}$ is $\mathcal{F}$-weak operator topology convergent and, for any $B \in \mathcal{F}$, the operator defined by $T_{B}^{*}(g)$ $=\sum_{j \in B} T_{j}^{*} g$ is compact. 
(b) If $X^{*}$ does not have a copy of $l_{\infty}, \sum_{j} T_{j}$ is unconditionally convergent if and only if $\sum_{j} T_{j}$ is $\mathcal{F}$-weak operator topology convergent and, for any $B \in \mathcal{F}$, the operator defined by $T_{B}(x)=\sum_{j \in B} T_{j} x$ is compact.

Proof: Let us check that, for $x \in X$, the series $\sum_{j} T_{j} x$ is unconditionally convergent in $Y$. If $\sum_{j} T_{j} x$ is not unconditionally convergent, then there exist $\varepsilon>0$ and a sequence $\left(F_{k}\right)_{k}$ in $\phi_{0}(\mathbb{N})$ such that $\sup F_{k}<\inf F_{k+1}$ and $\left\|\sum_{j \in F_{k}} T_{j} x\right\|>\varepsilon$, for $k \in \mathbb{N}$. We can assume that $Y$ is separable and we have that, for $k \in \mathbb{N}$, there exists $f_{k} \in S_{Y}$. such that $f_{k}\left(\sum_{j \in F_{k}} T_{j} x\right)>\varepsilon$. We can also assume that there exists $f_{0} \in B_{Y^{*}}$ such that $w^{*} \lim _{k} f_{k}=f_{0}$. For $j \in \mathbb{N}$, let us define the finitely additive measure $\mu_{j}: \mathcal{F} \rightarrow \mathbb{R}$ by $\mu_{j}(B)=f_{j}\left(w \sum_{j \in B} T_{j} x\right)$. We have that, for $B \in \mathcal{F}, \lim _{j} \mu_{j}(B)=f_{0}\left(w \sum_{j \in B} T_{j} x\right)$. Since $\mathcal{F}$ has the Vitali-Hahn-Saks property, $\left(\mu_{j}\right)_{j}$ is strongly uniformly additive. Therefore if $\left(B_{i}\right)_{i}$ is a disjoint sequence in $\mathcal{F}$ then $\lim _{i} \mu_{j}\left(B_{i}\right)=0$ uniformly in $j \in \mathbb{N}$. This contradicts the fact that $\mu_{k}\left(F_{k}\right)=f_{k}\left(\sum_{j \in F_{k}} T_{j} x\right)>\varepsilon$, for $k \in \mathbb{N}$.

It can also be proved, as before, that the series $\sum_{j} g T_{j}$ is unconditionally convergent in $X^{*}$ for every $g \in Y^{*}$.

If $\sum_{j} T_{j}$ is not unconditionally convergent then, a proof similar to Theorem 2.1 shows that the matrix $\left(g_{i} T_{j}\right)_{i, j}$ in $X^{*}$ satisfies the following.

(i) $\sum_{j} g_{i} T_{j}$ is unconditionally convergent, for $i \in \mathbb{N}$;

(ii) the sequence $\left(g_{i} T_{j}\right)_{i}$ is convergent, for $j \in \mathbb{N}$;

(iii) the sequence $\left(\sum_{j \in B} g_{i} T_{j}\right)_{i}$ is convergent, for $B \in \mathcal{F}$;

(iv) there exist $\varepsilon>0$ and a disjoint sequence $\left(F_{k}\right)_{k}$ in $\phi_{0}(\mathbb{N})$ such that $\left\|\sum_{j \in F_{k}} g_{k} T_{j}\right\|>\varepsilon$, for $k \in \mathbb{N}$.

For $i \in \mathbb{N}$ let us consider the vector measure $\mu_{i}: \mathcal{F} \rightarrow X^{*}$ defined by $\mu_{i}(B)$ $=\sum_{j \in B} g_{i} T_{j}$, for $B \in \mathcal{F}$. It is clear that every $\mu_{i}$ is $\sigma$-additive and that $\left(\mu_{i}\right)_{i}$ is pointwise convergent in $\mathcal{F}$. Therefore, $\left(\mu_{i}\right)_{i}$ is uniformly strongly additive in $\mathcal{F}$. This contradicts that $\left\|\mu_{i}\left(F_{i}\right)\right\|>\varepsilon$ for $i \in \mathbb{N}$.

REMARK. As a consequence of Corollary 2.2, by considering $\mathcal{F}=\mathcal{P}(\mathbb{N})$, we can prove Kalton's theorem (see the introduction). If $\mathcal{F}$ is a natural family that is subsequentially complete, we could use the proof of Kalton to obtain the corresponding results. However, we now prove that there exist natural families with the property $S_{1}$ that are not subsequentially complete.

Let $B_{1}$ be the family of the subsets $A \subset \mathbb{N}$ such that $A$ and $A^{c}$ have infinite even numbers and infinite odd numbers. Let $\mathcal{F}=B_{1} \cup \phi_{0}(\mathbb{N})$. We first prove that $\mathcal{F}$ is not subsequentially complete. Let us consider the sequence $(\{2 n\})_{n \in \mathbb{N}}$. It is easy to check that the union of the terms of any subsequence is not in $\mathcal{F}$. Hence, $\mathcal{F}$ is not subsequentially 
complete. It is easy to check that $\mathcal{F}$ has property $S_{1}$.

REMARK. On the conditional convergence of series in $K(X, Y)$. Ronglu and Kang [9] considered several conditions for the conditional convergence of a series and for the convergence of double series and iterated series.

We say that a natural family $\mathcal{F}$ has property $P_{1}([3])$ if there exists a map $f: \mathbb{N} \rightarrow \mathbb{N}$ such that for any pair $\left(F_{r}\right)_{r},\left(m_{r}\right)_{r}$ of sequences in $I_{0}(\mathbb{N})$ (the family of finite intervals of $\mathbb{N}$ ) and $\mathbb{N}$, respectively, with $m_{r} \leqslant \inf F_{r} \leqslant \sup F_{r}<m_{r+1}$ for $r \in \mathbb{N}$, there exist $B \in \mathcal{F}$ and an infinite set $M \subseteq \mathbb{N}$ that:

(a) $B \cap\left[m_{r}, m_{r+1}\right)=F_{r}$ for $r \in M$, and

(b) $B \cap\left(m_{r}, m_{r+1}\right)$ is either empty, or for $r \in \mathbb{N} \backslash M$ and $r>1$, it can be written as the union of at most $f(r-1)$ intervals.

It has been proved, in [3], that if $\mathcal{F}$ is a natural family with the property $P_{1}$ and $\left(x_{i j}\right)_{i, j}$ is a matrix in a Banach space $X$ such that the sequence $\left(\sum_{j \in B} x_{i j}\right)_{i}$ is convergent,
for $B \in \mathcal{F}$, then:

(i) $\sum_{j} x_{i j}$ is convergent, for $i \in \mathbb{N}$;

(ii) $\lim _{i}\left(\sum_{j} x_{i j}\right)=\sum_{j} x_{j}$, where $x_{j}=\lim _{i} x_{i j}$ for $j \in \mathbb{N}$; and

(iii) $\lim _{i} \sum_{j \in F} x_{i j}=\sum_{j \in F} x_{j}$ uniformly in the sets $F$ of the family $I_{0}(\mathbb{N})$. If, moreover, the series $\sum_{i}\left(\sum_{j \in B} x_{i j}\right)$ is convergent, for any $B \in \mathcal{F}$, then $\sum_{i} \sum_{j} x_{i j}=\sum_{j} \sum_{i} x_{i j}$.

As a consequence of the results that appear in the former remark and by proceeding as in the proof of Theorem 2.1, the following result can be proved:

THEOREM 2.4. Let $X, Y$ be two Banach spaces and let $\mathcal{F}$ be a natural family with the property $P_{1}$. Let $\left(T_{j}\right)_{j}$ be a sequence in $K(X, Y)$ such that, for each $B \in \mathcal{F}$,

(i) $\sum_{j \in B} T_{j}$ is stronger operator topology convergent,

(ii) $\sum_{j \in B} T_{j}^{*}$ is stronger operator topology convergent, and

(iii) the operator $T_{B}$ defined by $T_{B}(x)=\sum_{j \in B} T_{j} x$ is compact. Then $\sum_{j} T_{j}$ is convergent in $K(X, Y)$.

In the former theorem we can replace hypothesis (ii) by the two following hypothesis:

(ii') the space $X^{*}$ does not have a copy of $l_{\infty}$, and

(ii") the family $\mathcal{F}$ is such that any real and $\mathcal{F}$-convergent series $\sum_{j} a_{j}$ is unconditionally convergent.

\section{Series weak unconditionally Cauchy in $C \mathcal{L}(X, Y)$}

Let $S$ be a subspace of $l_{\infty}$ such that $c_{0} \subset S$ and let $X$ be a Banach space. We denote by $X(S)([1])$ the space of the sequences $\left(x_{i}\right)_{i} \in X^{\mathrm{N}}$ such that $\sum_{i} a_{i} x_{i}$ converges 
for $\left(a_{i}\right)_{i} \in S$. It is clear that $X(S)$ is a Banach space with the norm

$$
\left\|\left(x_{i}\right)_{i}\right\|=\sup \left\{\sum_{i=1}^{n}\left|f\left(x_{i}\right)\right|: n \in \mathbb{N}, f \in S_{X^{*}}\right\}=\sup \left\{\left\|\sum_{i=1}^{\infty} a_{i} x_{i}\right\|:\left(a_{i}\right)_{i} \in B_{C_{00}}\right\} .
$$

With this notation, we have that $X\left(c_{0}\right)$ can be identified to the space of weak unconditionally Cauchy series in $X$ (it can also be identified with the space $l_{1}^{w}(X)$ of the weakly 1- summing sequences [6]). The space $X\left(l_{\infty}\right)$ can be identified with the space of unconditionally convergent series.

We denote by $c(X)$ the space of the convergent sequences $\left(x_{i}\right)_{i} \in X^{\mathbf{N}}$, endowed with the sup norm. Similarly, $c_{0}(X)$ will denote the subspace of the sequences $\left(x_{i}\right)_{i} \in c(X)$ such that $\lim _{i} x_{i}=0$.

Our next theorem provides a simple characterisation of weak unconditionally Cauchy series in $C \mathcal{L}(X, Y)$.

TheOREM 3.1. Let $X, Y$ be two Banach spaces and let $\sum_{j} T_{j}$ be a series in $C \mathcal{L}(X, Y)$. Then, $\sum_{j} T_{j}$ is weak unconditionally Cauchy if and only if, for any sequence $\left(x_{i}\right)_{i} \in c(X)$ such that $\lim _{i} x_{i}=x_{0},\left(T_{j} x_{i}\right)_{j} \in Y\left(c_{0}\right)$ for $i \in \mathbb{N}$ and the sequence $\left(\left(T_{j} x_{i}\right)_{j}\right)_{i}$ converges to $\left(T_{j} x_{0}\right)_{j}$.

PROOF: Let us suppose that $\sum_{j} T_{j}$ is a weak unconditionally Cauchy series. Let us denote by $H=\left\|\left(T_{j}\right)_{j}\right\|$ the norm of $\left(T_{j}\right)_{j}$ in the space of weak unconditionally Cauchy series in $C \mathcal{L}(X, Y)$. For $x \in B_{X}$ and $g \in B_{Y *}$, the map $\varphi_{x g}: C \mathcal{L}(X, Y) \rightarrow \mathbb{R}$ defined by $\varphi_{x g}(T)=g(T x)$ is linear, continuous and satisfies $\left\|\varphi_{x g}\right\| \leqslant 1$. Therefore, $\sum_{j=1}^{\infty}\left|g T_{j}(x)\right| \leqslant H$.

If $M \subset \mathbb{N}$ is a finite set, then $\left\|\sum_{j \in M} g T_{j}\right\| \leqslant H$. Let $\left(x_{i}\right)_{i} \in c(X)$ and let $x_{0}=\lim _{i} x_{i}$. It is clear that $\vec{y}^{i}=\left(T_{j} x_{i}\right)_{j}$ is an element of $Y\left(c_{0}\right)$ for every $i \in \mathbb{N}$. For any given $\varepsilon>0$ there exists an $n_{0} \in \mathbb{N}$ such that $\left\|x_{p}-x_{q}\right\| \leqslant \varepsilon / H$ for $p, q \geqslant n_{0}$.

If $p, q \geqslant n_{0}$, we have that there exist $g \in S_{Y^{*}}$ and $n \in \mathbb{N}$ such that

$$
\left\|\bar{y}^{p}-\bar{y}^{q}\right\|-\varepsilon / 2<\sum_{j=1}^{n}\left|g T_{j}\left(x_{p}\right)-g T_{j}\left(x_{q}\right)\right| \leqslant\left\|x_{p}-x_{q}\right\|\left\|\sum_{j=1}^{n} g T_{j}\right\| \leqslant \varepsilon .
$$

Therefore, the sequence $\left(\bar{y}^{i}\right)_{i}$ is convergent in $Y\left(c_{0}\right)$ and $\lim _{i} \bar{y}^{i}=\bar{y}^{0}$ in $Y\left(c_{0}\right)$, where $\bar{y}^{0}=\left(T_{j} x_{0}\right)_{j}$.

In order to prove sufficiency, let us suppose $\sum_{j} T_{j}$ is not a weak unconditionally Cauchy series. We can inductively obtain a sequence $\left(F_{n}\right)_{n} \subset \phi_{0}(\mathbb{N})$ such that $\sup F_{n}$ $<\inf F_{n+1}$ and $\left\|\sum_{j \in F_{n}} T_{j}\right\|>2^{n}$. For $n \in \mathbb{N}$ there exists a $x_{n} \in S_{X}$ such that $\left\|\sum_{j \in F_{n}} T_{j} x_{n}\right\|$ $>2^{n}$. Let $y_{n}=\left(1 / 2^{n}\right) x_{n}$, for $n \in \mathbb{N}$. Then $\lim \left\|y_{n}\right\|=0$ and, by our hypothesis, $\left(\left(T_{j} y_{i}\right)_{j}\right)_{i}$ is a sequence in $Y\left(c_{0}\right)$ that converges to zero. This contradicts that $\left\|\sum_{j \in F_{n}} T_{j} y_{n}\right\|>1$ for $n \in \mathbb{N}$. 
Remark. (a) Let $X, Y$ be two Banach spaces and let $\sum_{j} T_{j}$ be a series in $C \mathcal{L}(X, Y)$. From the proof of theorem 3.1, it can be deduced that:

(i) $\sum_{j} T_{j}$ is weak unconditionally Cauchy if and only if $\left(\left(T_{j} x_{i}\right)_{j}\right)_{i}$ converges to zero in $Y\left(c_{0}\right)$, for any unconditionally convergent series $\sum_{i} x_{i}$ in $X$;

(ii) $\sum_{j} T_{j}$ is weak unconditionally Cauchy if and only if $\left(\left(T_{j} x_{i}\right)_{j}\right)_{i}$ converges to zero in $Y\left(c_{0}\right)$, for any absolutely convergent series $\sum_{i} x_{i}$ in $X$.

(b) When $Y=\mathbb{R}$ and $\sum_{j} f_{j}$ is a series in $X^{*}$, we have that $\sum_{j} f_{j}$ is weak unconditionally Cauchy if and only if for any sequence $\left(x_{i}\right)_{i} \in c(X),\left(\left(f_{j}\left(x_{i}\right)\right)_{j}\right)_{i}$ is convergent in $\mathbb{R}\left(c_{0}\right)$ (the space of unconditionally convergent series in $\mathbb{R}$ ).

Our next result provides another type of characterisation of weak unconditionally Cauchy series in $C \mathcal{L}(X, Y)$, when $Y$ satisfies additional properties.

ThEOREM 3.2. Let $X, Y$ be two Banach spaces and let us suppose that $Y^{*}$ has the Dunford-Pettis property. Let $\sum_{j} T_{j}$ be a series in $C \mathcal{L}(X, Y)$. Then, $\sum_{j} T_{j}$ is weak unconditionally Cauchy if and only if for any two weakly convergent sequences $\left(x_{i}\right)_{i}$ in $X$ and $\left(g_{i}\right)_{i}$ in $Y^{*}$, to $x_{0}$ and $g_{0}$ respectively, we have that $\left(g_{i} T_{j} x_{i}\right)_{j} \in c$ for $i \in \mathbb{N}$ and $\left(\left(g_{i} T_{j} x_{i}\right)_{j}\right)_{i}$ converges to $\left(g_{0} T_{j} x_{0}\right)_{j}$.

Proof: Let us suppose that $\sum_{j} T_{j}$ is weak unconditionally Cauchy. Let $\left(x_{i}\right)_{i},\left(g_{i}\right)_{i}$ be two sequences, in $X$ and $Y^{*}$ respectively, such that $w \lim _{i} x_{i}=x_{0}$ and $w \lim _{i} g_{i}=g_{0}$. Let us consider the matrix $\left(g_{i} T_{j} x_{i}\right)_{i, j}$. We have that any row defines an unconditionally convergent series and the $j$ th column converges to $g_{0} T_{j} x_{0}$, for $j \in \mathbb{N}$. For $M \subset \mathbb{N}$, we consider the map $T_{M}: X \rightarrow Y^{* *}$ defined by $T_{M}(x)=w^{*} \sum_{j \in M} T_{j} x$. This map is linear and continuous and we have that $w \lim _{i}\left(w^{*} \sum_{j \in M} T_{j} x_{i}\right)=w^{*} \sum_{j \in M} T_{j} x_{0}$. Since $Y^{*}$ is DunfordPettis property and $w \lim g_{i}=g_{0}, \lim _{i} \sum_{j \in M}^{j \in M} g_{i} T_{j} x_{i}=\sum_{j \in M}^{j \in M} g_{0} T_{j} x_{0}$. This proves that the matrix $\left(g_{i} T_{j} x_{i}\right)_{i, j}$ satisfies the conditions to be a basic matrix [4]. As a consequence, the sequence $\left(\left(g_{i} T_{j} x_{i}\right)_{j}\right)_{i}$ converges in $c$ to $\left(g_{0} T_{j} x_{0}\right)_{j}$.

Conversely, let us suppose that the series $\sum_{j} T_{j}$ is not weak unconditionally Cauchy. We can obtain a sequence $\left(F_{k}\right)_{k}$ in $\phi_{0}(\mathbb{N})$ such that $\sup F_{k}<\inf F_{k+1}$ and $\left\|\sum_{j \in F_{k}} T_{j}\right\|>2^{2 k}$ for $k \in \mathbb{N}$. For $k \in \mathbb{N}$, there exist $x_{k} \in S_{X}$ and $g_{k} \in S_{Y^{*}}$ such that $\left\|\sum_{j \in F_{k}} g_{k} T_{j} x_{k}\right\|$ $>2^{2 k}$ for $k \in \mathbb{N}$. We have that $\lim \left(1 / 2^{k}\right) g_{k}=0$ and $\lim \left(1 / 2^{k}\right) x_{k}=0$. We can easily obtain a contradiction, because of the convergence of the sequence $\left(\left(\left(1 / 2^{k}\right) g_{i} T_{j}\left(1 / 2^{k}\right) x_{i}\right)_{j}\right)_{i}$. REMARK 3.4. Let $X, Y$ be two Banach spaces. If $X$ has the Dunford-Pettis property and does not have a copy of $l_{1}$, or $Y$ is subsequentially complete, then $K(X, Y)$ $=W K(X, Y)$ (the space of weakly compact maps) and the results of this section remain valid if we replace the term " compact operator" by the term "weakly compact operator" We do not know any result for weakly compact operators similar to Kalton's theorem. 


\section{REFERENCES}

[1] A. Aizpuru and F.J. Pérez-Fernández, 'Spaces of $S$-Bounded multiplier convergent series', Acta Math. Hungar. 87 (2000), 135-146.

[2] A. Aizpuru and A. Gutiérrez-Dávila, 'Unconditionally Cauchy series and uniform convergence on matrices', (Preprint, 2001).

[3] A. Aizpuru and A. Gutiérrez-Dávila, 'On the interchange of series and some applications', Bull. Belg. Math. Soc. (to appear).

[4] P. Antosik and C. Swartz, Matrix methods in analysis, Lectures notes in Mathematics 1113 (Springer-Verlag, Berlin, Heidelberg, New York, 1985).

[5] Q. Bu and C. Wu, 'Unconditionally convergent series of operators on Banach spaces', $J$. Math. Anal. Appl. 207 (1997), 291-299.

[6] J. Diestel, H. Jarchow and A. Tonge, Absolutely summing operators, Cambridge studies in advanced mathematics 43 (Cambridge University Press, Cambridge, 1995).

[7] V. Kaftal and G. Weiss, 'A Riemann type theorem for unconditional convergence of operators', Proc. Amer. Math. Soc. 98 (1986), 431-435.

[8] N.J. Kalton, 'Spaces of compact operators', Math. Ann. 208 (1974), 267-278.

[9] L. Ranglu and S.M. Kang, 'On convergence of double series', J. Math. Anal. Appl. 240 (1999), 481-490.

[10] W. Schachermayer, 'On some classical Measure theoretic theorems for non $\sigma$-complete boolean algebras', Tecnical report, Linz University, Linz, 1978.

Departamento de Matemáticas

Universidad de Cádiz

Apdo. 40

11510-Puerto Real (Cádiz)

Spain

e-mail: antonio.aizpuru@uca.es

antonio.gutierrez@uca.es

antonio.sala@uca.es 